\title{
Association of TNFAIP3 Polymorphism with Susceptibility to Systemic Lupus Erythematosus in a Japanese Population
}

\author{
Aya Kawasaki, ${ }^{1}$ Ikue Ito, ${ }^{1}$ Satoshi Ito, ${ }^{2}$ Taichi Hayashi, ${ }^{2}$ Daisuke Goto, ${ }^{2}$ Isao Matsumoto, ${ }^{2}$ \\ Yoshinari Takasaki, ${ }^{3}$ Hiroshi Hashimoto, ${ }^{4}$ Takayuki Sumida, ${ }^{2}$ and Naoyuki Tsuchiya ${ }^{1}$ \\ ${ }^{1}$ Doctoral Program in Life System Medical Sciences, Graduate School of Comprehensive Human Sciences, University of Tsukuba, \\ 1-1-1 Tennodai, Tsukuba 305-8575, Japan \\ ${ }^{2}$ Division of Clinical Immunology, Doctoral Program in Clinical Sciences, Graduate School of Comprehensive Human Sciences, \\ University of Tsukuba, 1-1-1 Tennodai, Tsukuba 305-8575, Japan \\ ${ }^{3}$ Division of Rheumatology, Department of Internal Medicine, Juntendo University, 2-1-1 Hongo, Bunkyo-ku, Tokyo 113-8421, Japan \\ ${ }^{4}$ School of Medicine, Juntendo University, 2-1-1 Hongo, Bunkyo-ku, Tokyo 113-8421, Japan
}

Correspondence should be addressed to Naoyuki Tsuchiya, tsuchiya-tky@umin.ac.jp

Received 13 December 2009; Accepted 11 March 2010

Academic Editor: Charles Via

Copyright ( 2010 Aya Kawasaki et al. This is an open access article distributed under the Creative Commons Attribution License, which permits unrestricted use, distribution, and reproduction in any medium, provided the original work is properly cited.

\begin{abstract}
Recent genome-wide association studies demonstrated association of single nucleotide polymorphisms (SNPs) in the TNFAIP3 region at 6q23 with systemic lupus erythematosus (SLE) in European-American populations. In this study, we investigated whether SNPs in the TNFAIP3 region are associated with SLE also in a Japanese population. A case-control association study was performed on the SNPs rs13192841, rs2230926, and rs6922466 in 318 Japanese SLE patients and 444 healthy controls. Association of rs2230926 $G$ allele with SLE was replicated in Japanese (allelic association $P=.033$, odds ratio [OR] 1.47, recessive model $P=.023$, OR 8.52). The association was preferentially observed in the SLE patients with nephritis. When the TNFAIP3 mRNA levels of the HapMap samples were examined using GENEVAR database, the presence of TNFAIP3 rs2230926 G allele was associated with lower mRNA expression of TNFAIP3 $(P=.013)$. These results indicated that TNFAIP3 is a susceptibility gene to SLE both in the Caucasian and Asian populations.
\end{abstract}

\section{Introduction}

Nuclear factor- $\kappa \mathrm{B}(\mathrm{NF}-\kappa \mathrm{B})$ is a transcription factor that regulates expression of various genes including pro-inflammatory cytokines. TNFAIP3 (tumor necrosis factor alpha-induced protein 3) gene codes for A20, a ubiquitin-modifying protein required to inhibit NF- $\kappa \mathrm{B}$ activation by several signaling pathways including TNF and Toll-like receptors [1]. TNFAIP3-deficient mice exhibited multiple organ inflammation and damage involving kidneys and joints [2]. NF- $\kappa \mathrm{B}$ induces expression of A20; thus, A20 plays a role in a negative feedback mechanism in NF- $\kappa$ B pathway.

The lupus phenotypes in Fcy receptor IIb-deficient mice were reduced by treatment with NF- $\kappa \mathrm{B}$ inhibitors [3], suggesting a role of NF- $\kappa \mathrm{B}$ activation in the pathogenesis of systemic lupus erythematosus (SLE). A recent study reported that A20 negatively regulates immunostimulatory effects of dendritic cells and attenuates antigen presentation [4]. Thus, reduced function of A20 may induce excessive activation of dendritic cells and lead to the loss of tolerance to selfantigens.

Since a genome-wide association study (GWAS) conducted by the Wellcome Trust Case Control Consortium reported association of TNFAIP3 region single nucleotide polymorphisms (SNPs) with rheumatoid arthritis (RA) and Crohn's disease in 2007 [5], association of SNPs in the region surrounding TNFAIP3 at $6 \mathrm{q} 23$ has been reported in several autoimmune diseases. Association of the TNFAIP3 region has subsequently been replicated in RA $[6,7]$ and was also reported in SLE by two groups using GWAS $[8,9]$. These observations implicated that TNFAIP3 may be a common susceptibility gene to multiple autoimmune diseases.

Specifically, Musone et al. reported independent association of three SNPs in the TNFAIP3 region, rs13192841, 
rs2230926, and rs6922466, with SLE in a EuropeanAmerican population [8]. The SNP rs2230926 is located in the coding region of TNFAIP3 and introduces amino acid substitution of Phe to Cys at position 127 in the ovarian tumor domain, which was suggested to participate in the inhibitory function of A20 through deubiquitination of adaptor proteins [1]. Cys127 allele product was modestly less effective in inhibiting NF- $\kappa$ B activation by TNF than Phe127 [8]. Recently, an extensive meta-analysis and imputation study by Bates et al. [10] demonstrated that the strongest association was detected at an intron 2 SNP rs5029939, which was originally detected by Graham et al. [9] and was in tight linkage disequilibrium (LD) with rs2230926. They also showed that the carrier of the risk allele was at higher risk of developing nephritis and hematological manifestations [10]. SNPs rs13192841 and rs6922466 were mapped within $250 \mathrm{~kb}$ upstream and downstream of the TNFAIP3 region, respectively.

Recently, we demonstrated that IRF5 [11], STAT4 [12], and $B L K[13]$ are associated with SLE not only in the Caucasians, but also in the Japanese. In this study, we carried out a case-control association study of the SNPs in the TNFAIP3 region to examine whether TNFAIP3 is involved in genetic predisposition to SLE also in Japanese.

\section{Patients and Methods}

2.1. Patients and Controls. A case-control association study was conducted in 318 Japanese patients with SLE (19 males and 299 females, mean \pm SD age $41.4 \pm 13.5$ years) and 444 healthy controls (219 males and 225 females, mean \pm SD age $34.0 \pm 9.9$ years), recruited at the University of Tsukuba, Juntendo University, and the University of Tokyo. All patients and healthy individuals were native Japanese living in the central part of the Japan. All patients met the criteria for SLE proposed by the American College of Rheumatology [14].

In addition to the originally recruited controls, genotype data of 934 Japanese healthy controls were retrieved from the "JSNP550typed" dataset in the JSNP DATABASE (http:// snp.ims.u-tokyo.ac.jp/jsnp_gtype_access.html) and analyzed [15].

This study was reviewed and approved by the research ethics committees of University of Tsukuba, Juntendo University, and the University of Tokyo. Informed consent was provided by all donors.

2.2. DNA Samples. Genomic DNA was extracted from peripheral blood leucocytes using QIAamp blood kit (QIAGEN, Hilden, Germany) and amplified using the GenomiPhi DNA Amplification Kit (Amersham Biosciences, Piscataway, NJ, USA), following the manufacturer's instructions. Whole genome amplification products were used for genotyping.

2.3. Genotyping. Genotyping of SNPs was carried out using the TaqMan genotyping assay (Applied Biosystems, Foster City, CA), according to the manufacturer's instructions. The TaqMan probes used in this study were as follows: assay ID: C_1575524_10 (rs13192841), C_7701116_10 (rs2230926), and C_29431844_10 (rs6922466). Amplification was performed in a $10 \mu \mathrm{l}$ reaction volume containing $5 \mu \mathrm{l}$ of TaqMan Genotyping Master Mix and $0.125 \mu \mathrm{l}$ of TaqMan genotyping assay mix using 7300 Real-Time PCR System (Applied Biosystems). Thermal cycling conditions consisted of initial denaturation at $95^{\circ} \mathrm{C}$ for 10 minutes, followed by 40 cycles of $95^{\circ} \mathrm{C}$ for 15 seconds and $60^{\circ} \mathrm{C}$ for 1 minute.

Genotyping of 934 controls in the "JSNP550typed" dataset was performed using the Illumina HumanHap550 BeadChip (Illumina, Inc., San Diego, CA), as indicated in the JSNP DATABASE.

2.4. Association of TNFAIP3 SNP with TNFAIP3 mRNA Expression. Association of TNFAIP3 rs2230926 genotype with TNFAIP3 mRNA expression was examined using the mRNA data from lymphoblastoid cell lines derived from 44 JPT (the Japanese in Tokyo, Japan) and $45 \mathrm{CHB}$ (the Han Chinese in Beijing, China) HapMap individuals available from the database of the Gene Expression Variation (GENEVAR) project at the Wellcome Trust Sanger Institute (http://www.sanger.ac.uk/humgen/genevar/), as previously described [11]. Genotypes of rs2230926 in HapMap samples were available from HapMap database (http://www.hapmap .org/index.html.en). Because there was no subject with $\mathrm{G} / \mathrm{G}$ genotype, mRNA levels of TNFAIP3 were compared between rs2230926 G/T $(n=24)$ and T/T $(n=65)$ subjects.

2.5. Statistical Analysis. Association analyses were conducted by chi-square test using $2 \times 2$ contingency tables. When 1 or more of the variables in the contingency tables was 5 or less, Fisher's exact test was employed. Because this study intended to examine whether the association of TNFAIP3 is replicated in Japanese, correction for multiple testing was not applied and the uncorrected $P$ values are shown.

Based on the allele frequencies in Japanese, the sample size of this study (318 patients and 444 controls) had the power of $80 \%$ to detect association when the genotype relative risk is 1.55 (rs13192841), 1.65 (rs2230926), and 1.45 (rs6922466), respectively [16].

To examine LD between the SNPs in the TNFAIP3 region, LD parameters $\left(D^{\prime}\right.$ and $\left.r^{2}\right)$ were calculated using Haploview version 4.0 software (Broad Institute of MIT and Harvard, Cambridge, MA), based on data from the 444 healthy Japanese controls.

Difference in the mRNA expression between the rs2230926 G/T and T/T subjects in the HapMap subjects was analyzed by unpaired Student's $t$-test.

\section{Results and Discussion}

To examine whether TNFAIP3 is associated with SLE in a Japanese population, an association study of rs13192841, rs2230926, and rs6922466 was conducted in 318 Japanese patients with SLE and 444 healthy controls. As observed in Caucasians [8], LD was modest among the examined three SNPs in 444 healthy Japanese controls $\left(r^{2}: 0.00-0.11, D^{\prime}\right.$ : 0.04-0.40). On the other hand, the intron 2 SNP rs5029939, which showed the strongest association in the study of 
TABLE 1: Association of the TNFAIP3 region SNPs with SLE in a Japanese population.

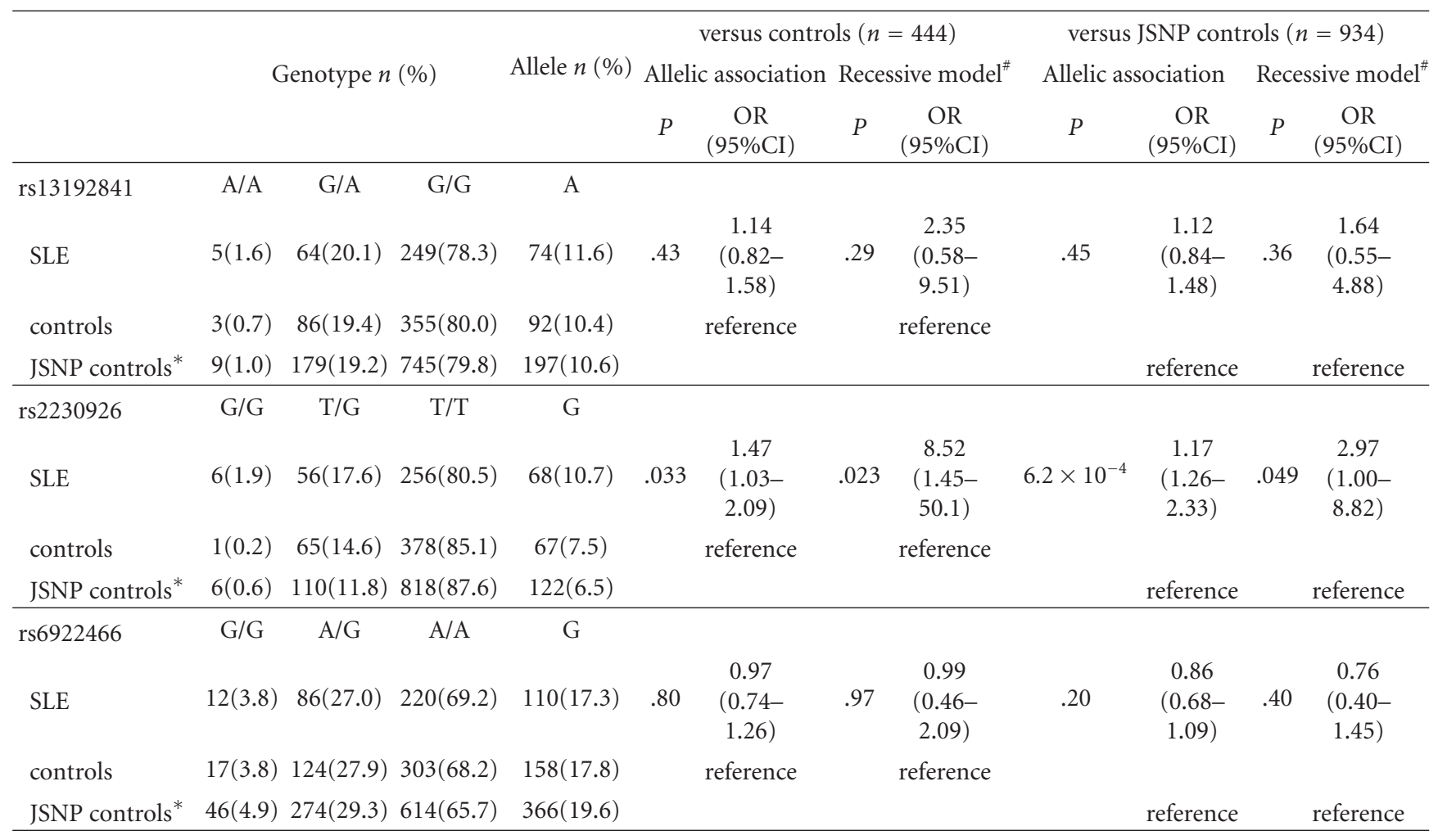

OR: odds ratio, $95 \%$ CI: confidence interval.

* Genotype data of healthy Japanese individuals obtained from JSNP DATABASE.

${ }^{*}$ Recessive model for the minor allele.

Bates et al. [10], was in strong LD with rs2230926 also in Japanese (HapMap JPT: $r^{2}=0.86, D^{\prime}=1.0$ ). None of the control genotypes were significantly deviated from HardyWeinberg equilibrium.

The association of rs2230926 with SLE was replicated in the Japanese, while evidence for association of rs13192841 and rs6922466 was not detected (Table 1). To confirm our findings, the patients' genotypes were also compared with the genotypes of 934 Japanese controls available from JSNP DATABASE. As shown in Table 1, rs2230926 G allele frequency was significantly increased in SLE patients compared with JSNP controls. On the other hand, association of rs13192841 and rs6922466 was not detected, even when the JSNP controls were used for comparison.

Because the minor allele frequency of rs6920220, previously shown to be associated both with RA and SLE in European-American populations $[6,7,9]$, was only 0.006 in the HapMap JPT samples, association was not examined for this SNP.

We next tested whether rs2230926 in TNFAIP3 was associated with the development of nephritis. Frequency of rs2230926 G allele was significantly increased in SLE with nephritis $(12.2 \%)$ as compared with healthy controls $(7.5 \%, P=.010$, OR $1.70,95 \%$ CI $1.13-2.56)$, while significant difference was not observed between SLE without nephritis (8.6\%) and healthy controls (Table 2). This finding is in agreement with Bates et al., who demonstrated preferential association of TNFAIP3 with SLE patients with nephritis in Caucasians [10]. Bates et al. also reported association of TNFAIP3 with hematological manifestations [10]. We did not carry out this analysis, however, because more than $90 \%$ of our patients fulfilled ACR classification criteria for hematologic disorder, especially lymphopenia $(1500 / \mu l)$.

A previous study suggested that the product of the risk allele, rs2230926 G (Cys127), may be modestly less efficient in inhibiting TNF-induced NF- $\kappa$ B activity [8]. To gain more insight into the functional significance of rs2230926, association of TNFAIP3 mRNA levels with genotype was evaluated using the HapMap JPT and CHB subjects. Data on the mRNA levels in B cell lines from HapMap samples were obtained from GENEVAR database (http://www.sanger.ac.uk/humgen/genevar/). As shown in Figure 1, the levels of TNFAIP3 mRNA were decreased in carriers of the rs2230926 G allele (G/T subjects) as compared with noncarriers $(\mathrm{T} / \mathrm{T})(P=.013)$. This finding is in agreement with a previous study which demonstrated that TNFAIP3 intronic SNP rs5029930, which is strong LD with rs2230926 G, was associated with lower expression of TNFAIP3 mRNA expression in peripheral mononuclear cells [17]. Thus, our findings suggested that the molecular mechanisms of association of TNFAIP3 haplotype containing rs2230926 G and rs5029939 G may involve both the functional change caused by the amino acid substitution at the protein level and the reduced expression level of A20 protein. 
TABLE 2: Association of TNFAIP3 rs2230926 with presence or absence of nephritis.

\begin{tabular}{|c|c|c|c|c|c|c|c|c|c|c|}
\hline \multirow{2}{*}{ rs2230926 } & \multicolumn{3}{|c|}{ Genotype } & \multirow{2}{*}{$\begin{array}{c}\text { Allele } \\
\text { G }\end{array}$} & \multicolumn{2}{|c|}{ Allelic association } & \multicolumn{2}{|c|}{ Recessive model ${ }^{*}$} & \multicolumn{2}{|c|}{ Dominant model $^{*}$} \\
\hline & $\mathrm{G} / \mathrm{G}$ & $\mathrm{T} / \mathrm{G}$ & $\mathrm{T} / \mathrm{T}$ & & $P$ & $\begin{array}{c}\text { OR } \\
(95 \% \mathrm{CI})\end{array}$ & $P$ & $\begin{array}{c}\text { OR } \\
(95 \% \mathrm{CI})\end{array}$ & $P$ & $\begin{array}{c}\text { OR } \\
(95 \% \mathrm{CI})\end{array}$ \\
\hline \multicolumn{11}{|l|}{ SLE } \\
\hline with nephritis $(n=168)$ & $4(2.4)$ & $33(19.6)$ & $131(78.0)$ & $41(12.2)$ & .010 & $\begin{array}{c}1.70 \\
(1.13-2.56)\end{array}$ & .022 & $\begin{array}{c}10.8 \\
(1.85-63.2)\end{array}$ & .035 & $\begin{array}{c}1.62 \\
(1.03-2.53)\end{array}$ \\
\hline without nephritis $(n=145)$ & $2(1.4)$ & $21(14.5)$ & $122(84.1)$ & $25(8.6)$ & .55 & $\begin{array}{c}1.16 \\
(0.72-1.87)\end{array}$ & .15 & $\begin{array}{c}6.20 \\
(0.75-51.1)\end{array}$ & .77 & $\begin{array}{c}1.08 \\
(0.64-1.81)\end{array}$ \\
\hline Controls $(n=444)$ & $1(0.2)$ & $65(14.6)$ & $378(85.1)$ & $67(7.5)$ & & reference & & reference & & reference \\
\hline
\end{tabular}

OR: odds ratio, 95\% CI: confidence interval. Parentheses in the genotype and allele frequency columns indicate percentages.

* Recessive and dominant models for the G allele.

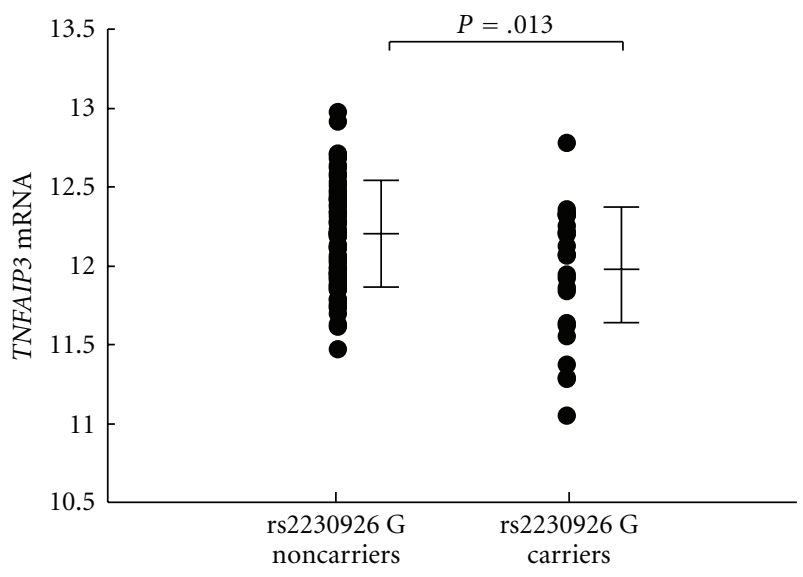

FIGURE 1: Association of TNFAIP3 rs2230926 with mRNA levels of TNFAIP3. Because there was no subject with G/G genotype, TNFAIP3 mRNA levels were compared between rs2230926 G/T $(n=$ 24 , mean \pm SD: $12.0 \pm 0.43)$ and $\mathrm{T} / \mathrm{T}(n=65$, mean \pm SD: $12.2 \pm$ 0.33 ) subjects by unpaired Student's $t$-test. The mRNA levels were obtained from the mRNA expression profiling data in the $\mathrm{B}$ cell lines from the HapMap JPT and CHB subjects (GENEVAR database).

A GWAS of SLE in a Chinese Han population was very recently reported for the first time in non-Caucasian populations [18]. In the study, association of TNFAIP3 rs2230926, but not rs13192841 and rs6922466, with SLE was demonstrated, which was consistent with our results in Japanese. Thus, taken together with previous observations, our study established TNFAIP3 as a susceptibility gene to SLE common to Caucasian and Asian populations. Of particular interest, both the GWAS in Chinese [18] and a large-scale study in Caucasians [19] identified association of TNFAIP3 interacting protein 1 (TNIP1), which binds to A20 and is thought to inhibit NF- $\kappa \mathrm{B}$ signaling. These findings underscore the crucial role of $\mathrm{NF}-\kappa \mathrm{B}$ regulation pathway in the genetic background of SLE in multiple populations.

\section{Conclusions}

The association of rs2230926 in the coding region of TNFAIP3 with SLE was replicated in a Japanese population. Our finding demonstrated that TNFAIP3 is a susceptibility gene to SLE both in the Asian and Caucasian populations.

\section{Acknowledgment}

This work was supported by Grant-in-Aid for Scientific Research (B) and Gran-in-Aid for Young Scientists (B) from Japan Society for the Promotion of Science (JSPS), Health and Labour Sciences Research Grants from the Ministry of Health, Labour and Welfare of Japan, and Mitsubishi Pharma Research Foundation.

\section{References}

[1] B. Coornaert, I. Carpentier, and R. Beyaert, "A20: central gatekeeper in inflammation and immunity," Journal of Biological Chemistry, vol. 284, no. 13, pp. 8217-8221, 2009.

[2] E. G. Lee, D. L. Boone, S. Chai, et al., "Failure to regulate TNFinduced NF-kappaB and cell death responses in A20-deficient mice," Science, vol. 289, no. 5488, pp. 2350-2354, 2000.

[3] A. M. Kalergis, M. I. Iruretagoyena, M. J. Barrientos, et al., "Modulation of nuclear factor-kappaB activity can influence the susceptibility to systemic lupus erythematosus," Immunology, vol. 128, no. 1, part 2, pp. e306-e314, 2009.

[4] X.-T. Song, K. Evel-Kabler, L. Shen, L. Rollins, X. F. Huang, and S.-Y. Chen, "A20 is an antigen presentation attenuator, and its inhibition overcomes regulatory $\mathrm{T}$ cell-mediated suppression," Nature Medicine, vol. 14, no. 3, pp. 258-265, 2008.

[5] The Wellcome Trust Case Control Consortium, "Genomewide association study of 14,000 cases of seven common diseases and 3,000 shared controls," Nature, vol. 447, no. 7145, pp. 661-678, 2007.

[6] W. Thomson, A. Barton, X. Ke, et al., "Rheumatoid arthritis association at 6q23," Nature Genetics, vol. 39, no. 12, pp. 1431$1433,2007$.

[7] R. M. Plenge, C. Cotsapas, L. Davies, et al., "Two independent alleles at 6q23 associated with risk of rheumatoid arthritis," Nature Genetics, vol. 39, no. 12, pp. 1477-1482, 2007.

[8] S. L. Musone, K. E. Taylor, T. T. Lu, et al., "Multiple polymorphisms in the TNFAIP3 region are independently associated with systemic lupus erythematosus," Nature Genetics, vol. 40, no. 9, pp. 1062-1064, 2008.

[9] R. R. Graham, C. Cotsapas, L. Davies, et al., "Genetic variants near TNFAIP3 on 6q23 are associated with systemic lupus erythematosus," Nature Genetics, vol. 40, no. 9, pp. 1059-1061, 2008.

[10] J. S. Bates, C. J. Lessard, J. M. Leon, et al., "Meta-analysis and imputation identifies a $109 \mathrm{~kb}$ risk haplotype spanning TNFAIP3 associated with lupus nephritis and hematologic 
manifestations," Genes and Immunity, vol. 10, no. 5, pp. 470477, 2009.

[11] A. Kawasaki, C. Kyogoku, J. Ohashi, et al., "Association of IRF5 polymorphisms with systemic lupus erythematosus in a Japanese population: support for a crucial role of intron 1 polymorphisms," Arthritis and Rheumatism, vol. 58, no. 3, pp. 826-834, 2008.

[12] A. Kawasaki, I. Ito, K. Hikami, et al., "Role of STAT4 polymorphisms in systemic lupus erythematosus in a Japanese population: a case-control association study of the STAT1STAT4 region," Arthritis Research and Therapy, vol. 10, no. 5, article R113, 2008.

[13] I. Ito, A. Kawasaki, S. Ito, et al., "Replication of the association between the C8orf13-BLK region and systemic lupus erythematosus in a Japanese population," Arthritis and Rheumatism, vol. 60 , no. 2, pp. 553-558, 2009.

[14] M. C. Hochberg, "Updating the American College of Rheumatology revised criteria for the classification of systemic lupus erythematosus," Arthritis and Rheumatism, vol. 40, no. 9, p. $1725,1997$.

[15] M. Hirakawa, T. Tanaka, Y. Hashimoto, M. Kuroda, T. Takagi, and Y. Nakamura, "JSNP: a database of common gene variations in the Japanese population," Nucleic Acids Research, vol. 30, no. 1, pp. 158-162, 2002.

[16] J. Ohashi, S. Yamamoto, N. Tsuchiya, et al., "Comparison of statistical power between $2 \times 2$ allele frequency and allele positivity tables in case-control studies of complex disease genes," Annals of Human Genetics, vol. 65, no. 2, pp. 197-206, 2001.

[17] W. Boonyasrisawat, D. Eberle, S. Bacci, et al., "Tag polymorphisms at the A20 (TNFAIP3) locus are associated with lower gene expression and increased risk of coronary artery disease in type 2 diabetes," Diabetes, vol. 56, no. 2, pp. 449-505, 2007.

[18] J.-W. Han, H.-F. Zheng, Y. Cui, et al., "Genome-wide association study in a Chinese Han population identifies nine new susceptibility loci for systemic lupus erythematosus," Nature Genetics, vol. 41, no. 11, pp. 1234-1237, 2009.

[19] V. Gateva, J. K. Sandling, G. Hom, et al., "A large-scale replication study identifies TNIP1, PRDM1, JAZF1, UHRF1BP1 and IL10 as risk loci for systemic lupus erythematosus," Nature Genetics, vol. 41, no. 11, pp. 1228-1233, 2009. 


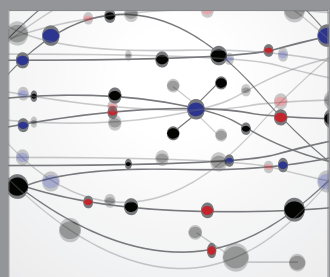

The Scientific World Journal
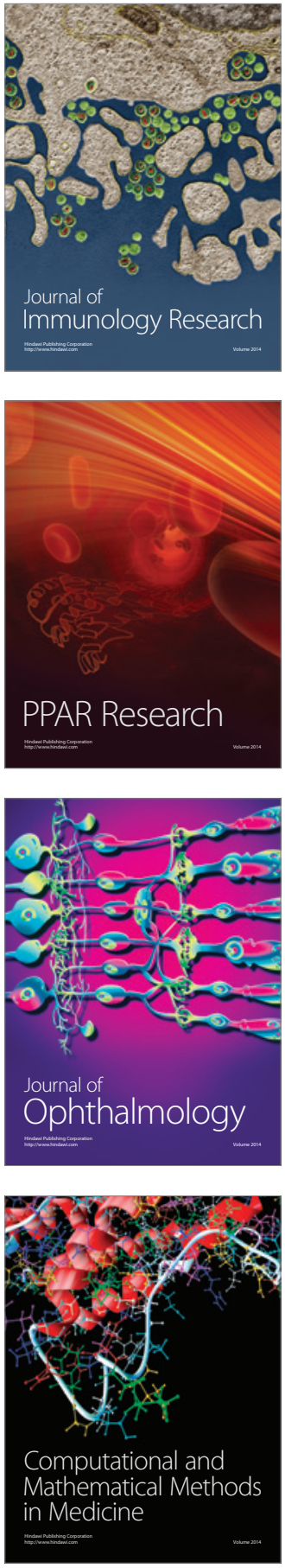

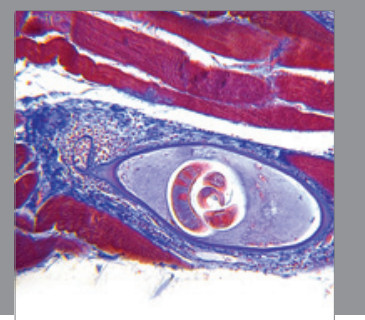

Gastroenterology

Research and Practice
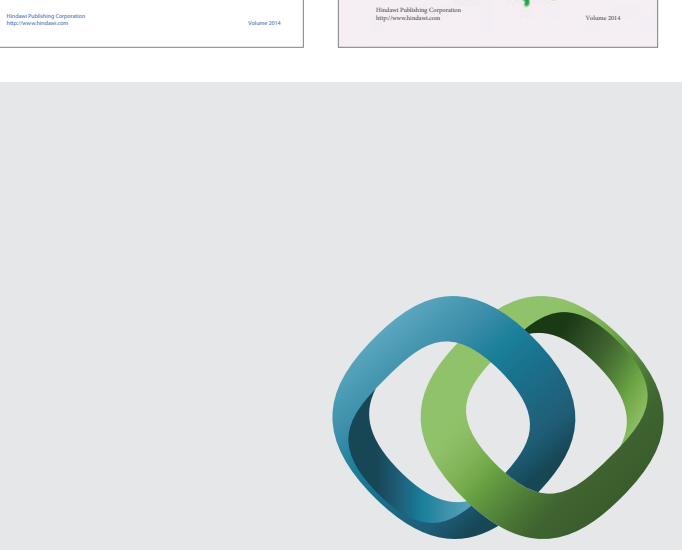

\section{Hindawi}

Submit your manuscripts at

http://www.hindawi.com
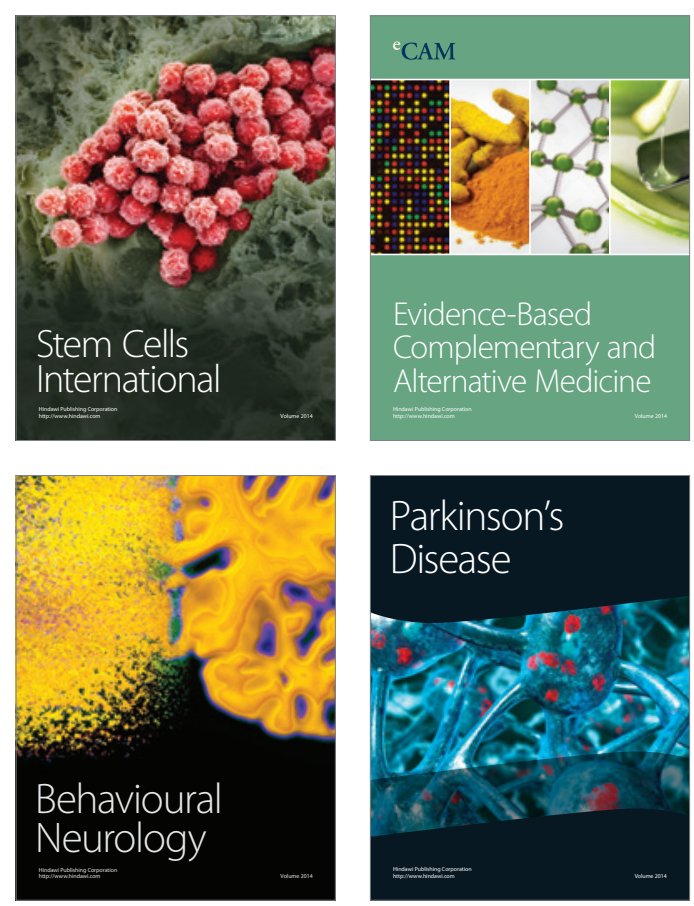

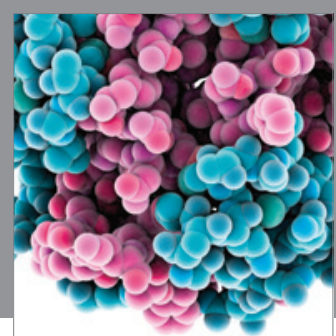

Journal of
Diabetes Research

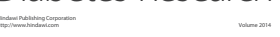

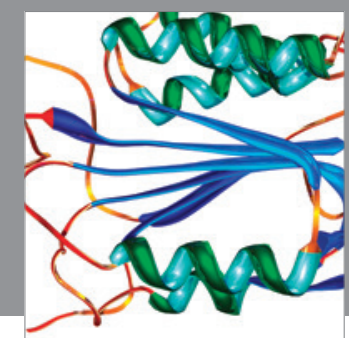

Disease Markers
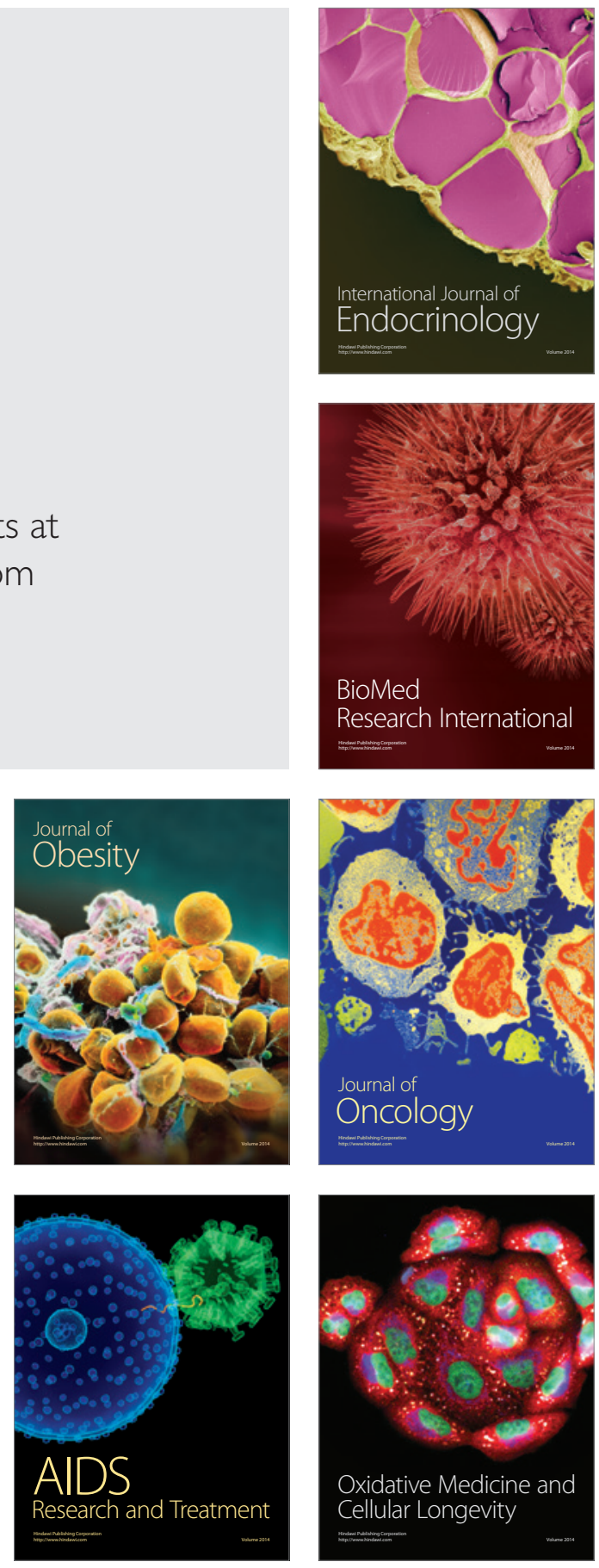